\title{
Acute Effects of Loaded Whole Body Vibration Training on Performance
}

\author{
Haris Pojskic ${ }^{1,2}$; Jeffrey Pagaduan ${ }^{3,{ }^{*}}$; Edin Uzicanin ${ }^{1,2}$; Fuad Babajic ${ }^{1,2}$; Melika Muratovic ${ }^{1,2}$; \\ Mario Tomljanovic ${ }^{4}$ \\ ${ }^{1}$ Department of Physical Education and Sport, Tuzla University, Tuzla, Bosnia and Herzegovina \\ ${ }^{2}$ Center for Sports Excellence, Tuzla, Bosnia and Herzegovina \\ ${ }^{3}$ College of Human Kinetics, University of the Philippines - Diliman, Quezon, Philippines \\ ${ }^{4}$ Faculty of Kinesiology, University of Split, Split, Croatia \\ *Corresponding author: Jeffrey Pagaduan, College of Human Kinetics, University of the Philippines-Diliman, Quezon City, Philippines. Tel:+63-29296033, E-mail: jcpagaduan@gmail.com
}

Received: October 4, 2014; Accepted: December 24, 2014

\begin{abstract}
Background: The application of whole body vibration (WBV) as a warm-up scheme has been receiving an increasing interest among practitioners.

Objectives: The aim of this study was to determine the effect of loaded and unloaded WBV on countermovement jump, speed and agility. Patients and Methods: Twenty-one healthy male college football players (age: $20.14 \pm 1.65$ years; body height: $179.9 \pm 8.34 \mathrm{~cm}$; body mass: $74.4 \pm 13.0 \mathrm{~kg}$; \% body fat: $9.45 \pm 4.8$ ) underwent randomized controlled trials that involved standing in a half squat position (ST), ST with $30 \%$ of bodyweight (ST $+30 \%$, whole body vibration at $\mathrm{f}=50 \mathrm{~Hz}, \mathrm{~A}=4 \mathrm{~mm}(\mathrm{WBV})$, and WBV with $30 \%$ bodyweight (WBV $+30 \% \mathrm{BW}$ ) after a standardized warm-up. Post measures of countermovement jump, 15-m sprint, and modified t-test were utilized for analyses.

Results: One way repeated measures ANOVA revealed a significant difference in the countermovement jump performance, $\mathrm{F}(3,60=9.06$, $\eta 2=2.21, \mathrm{P}=0.000$. Post-hoc showed that $\mathrm{WBV}+30 \% \mathrm{BW}$ posted significant difference compared to $(\mathrm{P}=0.008), \mathrm{ST}+30 \% \mathrm{BW}(\mathrm{P}=0.000)$ and $\operatorname{WBV}(\mathrm{P}=0.000)$. There was also a significant difference in the sprint times among interventions, $\mathrm{F}(3,60)=23.0, \eta 2=0.865, \mathrm{P}=0.000$. Post hoc showed that $\mathrm{WBV}+30 \% \mathrm{BW}$ displayed significantly lower time values than $\mathrm{ST}(\mathrm{P}=0.000), \mathrm{ST}+30 \% \mathrm{BW}(\mathrm{P}=0.000)$ and $\mathrm{WBV}(\mathrm{P}=$ 0.000 ). Lastly, there was a significant difference in the agility performance across experimental conditions at $F(2.01,40.1)=21.0, \eta 2=0.954$, $\mathrm{P}=0.000$. Post hoc demonstrated that $\mathrm{WBV}$ have lower times than ST $(\mathrm{P}=0.013)$. Also, $\mathrm{WBV}+30 \% \mathrm{BW}$ posted lower times compared to ST $(\mathrm{P}$ $=0.000), \mathrm{ST}+30 \%(\mathrm{P}=0.000)$ and $\mathrm{WBV}(\mathrm{P}=0.003)$.

Conclusions: Additional external load of $30 \%$ bodyweight under WBV posted superior gains in countermovement jump, speed and agility compared to unloaded WBV, loaded non-WBV and unloaded non-WBV interventions.
\end{abstract}

Keywords:Exercise; Warm-Up Exercise; Athletic Performance; Football

\section{Background}

Warm-up is generally promoted as an activity that improves athletes' performance. It is especially important in team sports demanding acceleration, deceleration, or change of direction (1). The general warm-up followed by different kinds of stretching exercises is commonly used. However, some coaches use complex or contrast loading in warm up sessions which involve strength training exercises (isometric or dynamic) followed by biomechanically similar plyometric exercise or vice versa (2). This concept is based on a phenomenon called postactivation potentiation (PAP) that is defined as an acute enhancement of muscular power output produced by performing a preload stimulus before an actual activity (3). It is known that any previous muscle activity can trigger both PAP and fatigue mechanisms (4). Contractile history of a muscle is said to have a positive effect on muscle performance especially in terms of twitch contractions, rate of force development and explosive movements (5).

Recently, there has been an increasing interest in the use of whole body vibration training (WBV) as a warm- up strategy. During WBV, mechanical vibrations produce compensatory muscle contractions as a result of tonic vibration reflex (TVR) via excitation of primary endings of muscle spindles and activation of alpha-motor neurons (6-8). Several acute studies revealed positive effects of WBV on physical performance (9-13). In contrast, other studies showed no beneficial effect of WBV on sprint (1417), jump $(10,15,17,18)$ and agility $(14,15,19)$ performance. The equivocal findings in literature may be explained by methodological differences applied in the studies.

Mechanical loading in WBV is one of the factors that can affect performance. Dabbs et al. (20) suggested that amplitudes between 4 and $10 \mathrm{~mm}$, durations of exposure ranging from 30 seconds to 4 minutes, a work to rest ratio of 1: 1-1:3, rest intervals, ranging from 0 to 10 minutes would be sufficient for optimal performance. Also, when frequency is $50 \mathrm{~Hz}$, amplitude should be around 4-6 mm. In a similar light, Ronnestad (21) proposed that additional external load during WBV may produce larger positive stimulus to the human body and power output especial-

Copyright (C) 2015. This is an open-access article distributed under the terms of the Creative Commons Attribution-NonCommercial 4.0 International License (http:/ creativecommons.org/licenses/by-nc/4.0/) which permits copy and redistribute the material just in noncommercial usages, provided the original work is properly cited. 
ly in well trained subjects. Another factor that can affect dose-response relationship in training schemes is the type of muscle contraction. Researchers suggested that utilization of a single-joint maximum voluntary isometric exercise (22) and a multi-joint isometric exercise (2325) elicit PAP.

\section{Objectives}

To the researchers' knowledge, no studies in warm-up settings were administered using WBV with prolonged intermittent low intensity isometric exercises. Such a void in the current literature failed to explain possible mechanisms of isometric contraction in WBV. Thus, the purpose of this study was to compare countermovement jump performance, speed, and agility after loaded and unloaded intermittent low intensity half-squat isometric exercise in vibration and non-vibration conditions in well trained soccer players. The researchers hypothesized that WBV with the extra load would acutely produce greatest gains in jump, sprint and agility in comparison with other preconditioning protocols.

\section{Patients and Methods}

\subsection{Subjects}

Twenty-one healthy male college football players (age: $20.14 \pm 1.65$ years; body height: $179.9 \pm 8.34 \mathrm{~cm}$; body mass: $74.4 \pm 13.0 \mathrm{~kg}$; \% body fat: $9.45 \pm 4.8$ ) from the Tuzla University with no history of neuromuscular disease or reported injuries for the past six months volunteered to participate in the study. Athletes trained 8 hours a week ( 4 sessions of 2 hours each) on field and 3 hours a week (2 sessions of 1.5 hours each) in the gym. Consumption of a light meal at least three hours prior to the beginning of testing sessions was allowed. Hydration taken in small amounts was also encouraged during testing sessions. Avoidance from strenuous activity, tobacco, alcohol consumption, caffeine intake, and sleep deprivation for at least 48 hours prior the testing sessions were also requested. Before the start of the study, subjects were informed about the potential benefits and risks associated with the study. A written informed consent was provided to all subjects. This study was approved by the Ethical Committee of the University of Tuzla, approved with procedures conforming to the principles of the Declaration of Helsinki on human experimentation.

\subsection{Procedures}

In this study, 5 experimentation sessions, separated by 48 hours, occurred at the Exercise Science Laboratory and in a sport hall of Faculty of Physical Education and Sport, Tuzla University from 8 a.m. to 10 a.m. at the early off-season training period. Day 1 was devoted to measurement of height, weight and percentage of body fat. Body height was measured to the nearest $0.01 \mathrm{~m}$ with a portable stadi- ometer (Astra scale 27310, Gima, Italy). Body weight(BW), body fat percentage was measured by a bioelectric body composition analyzer (Tanita TBF-300 increments $0.1 \%$; Tanita, Tokyo, Japan).

Randomized controlled trials were carried out in the remaining four sessions at which the subjects underwent standing in a static half squat position (ST), ST with $30 \%$ of bodyweight (ST $+30 \%$ ), whole body vibration at $\mathrm{f}=50$ $\mathrm{Hz}, \mathrm{A}=4 \mathrm{~mm}$ (WBV), and WBV with an additional load of $30 \%$ of subject's bodyweight (WBV $+30 \%$ BW). Each intervention was performed 5 times for 60 seconds with a rest interval of 30 seconds in between sets. The subjects stepped off the platform and stood for 30 seconds. The knee flexion angle at the static half squat position was approximately 100 degrees (checked by goniometer before the interventions) with the feet slightly wider then shoulder width apart and the heels on the floor. An Olympic $\operatorname{bar}(20 \mathrm{~kg})$ and appropriate weighted plates were used to equate a subject's external load of $30 \%$ of BW.

All experimental conditions were preceded by a general warm-up (GW) that consisted of five minutes running at a preset pace. This was equivalent to 12 circles around an $86 \mathrm{~m}$ circumference area. In the first four circles, the participants had to run 30 seconds per circle. 25 seconds retain was required to finish the second four circles. In the last four circles, the participants had to run $20 \mathrm{sec}$ onds per circle. After GW participants had one minute rest and carried on with dynamic active stretching (DS). DS consisted of 7 exercises performed in 7 minutes. Each exercise consisted of 2 sets of 20 seconds with a rest interval of 10 seconds between sets. The rest interval between exercises was 10 seconds. After DS participants had one minute rest before proceeding to one of the experimental protocols.

After 2 minutes of an intervention, the participants performed the countermovement jump test (CMJ), 15-m sprint test and the modified agility test. Each test was performed two times. Intratest and intertest rest interval was two minutes. Test-retest reliabilities were 0.96, 0.89, and 0.86 for CMJ, 15-m sprint test and the modified T agility test respectively. In the CMJ, the hands on waist CMJ protocol was used to eliminate the contribution of arm swing in jump performance. For testing the CMJ height, OptoJump System (Microgate, Bolzano, Italy) was used. The participants started in an upright position and executed a countermovement immediately before a jump. The participants were encouraged to land in an upright position, but to bend their knees after landing to reduce mechanical stress. For the $15-\mathrm{m}$ sprint test, the subjects sprinted from a stationary position located $1 \mathrm{~m}$ before an automated timing system (Speedtrap II, Brower Timing Systems, Draper, UT, USA). To avoid error, the laser beam was positioned so the height above the ground approximated the height of the subjects' waist. Once the subjects were prepared, they started on their own decision.

In the modified t-test, the subjects started $1 \mathrm{~m}$ behind an automated timing system (Speed trap II, Brower Tim- 
Pojskic Het al.

ing Systems, Draper, UT, USA) and carried out a 5-meter sprint. After the sprint, the participants shuffled to the left $(2.5 \mathrm{~m})$ and shuffled back to the right for $5 \mathrm{~m}$. This was continued by a $2.5 \mathrm{~m}$ shuffle to the left and back sprint for $5 \mathrm{~m}$. Once the athletes were prepared, they started on their own decision. For the 15-m sprint test and modified t-test, timing sensor was positioned at the height of the subjects' waist (Figure 1). All players were familiar with the testing procedures because they routinely performed the tests during individual strength and conditioning programs. The subjects were encouraged to make as much effort as possible during all tests. The best trial for each test was recorded for analysis. The experimental protocol is displayed on Figure 2.

\subsection{Statistical Procedures}

Data are displayed as means and standard deviations. Kolmogrov-Smirnov test showed normal distribution of data. One-way repeated measures ANOVA were utilized to determine significant difference in performance. Mauchly's test was used to examine the sphericity of data (26). Any violation in sphericity was corrected using the Greenhouse-Geisser estimates of sphericity (27). Eta squared ( $\eta 2$ ) was used to estimate effect size. Pairwise comparison was determined using Bonferonni post hoc contrast. Analyses were performed using a commercial software (SPSS Inc., Chicago, IL; Version 14.0) with alpha set at 0.05 level of significance.

\section{Results}

Mauchley's test for assumption of sphericity on CMJ data was met. Results from the one-way repeated measures ANOVA showed that there was a significant difference in CMJ across interventions, $F(3,60)=9.06, \eta 2$ $=2.21, \mathrm{P}=0.000$. Bonferonni post hoc showed that WBV $+30 \%$ BW was significantly higher compared to ST (P =
$0.008), \mathrm{ST}+30 \% \mathrm{BW}(\mathrm{P}=0.000)$ and $\mathrm{WBV}(\mathrm{P}=0.000)$. Figure 3 shows the effect of various experimental protocols on CMJ performance.

For the 15-m sprint, assumption of sphericity of data agreed with Mauchly's test. There was a significant difference in sprint time across interventions, $\mathrm{F}(3,60)=$ 23.0, $\eta=0.865, P=0.000$. Post hoc identified that WBV + $30 \% \mathrm{BW}$ posted significantly lower time values than ST (P $=0.000), \mathrm{ST}+30 \% \mathrm{BW}(\mathrm{P}=0.000)$ and WBV $(\mathrm{P}=0.000)$. The effect of experimental protocols on 15-m sprint is displayed on Figure 4.

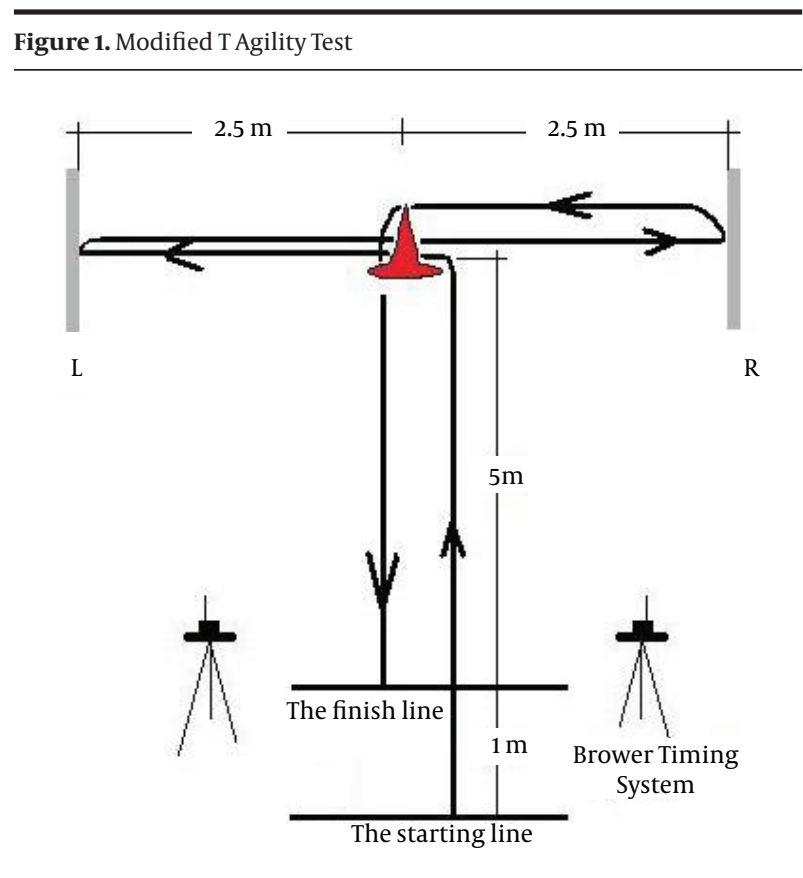

L: left line, R: right line

EXPERIMENTAL DESIGN

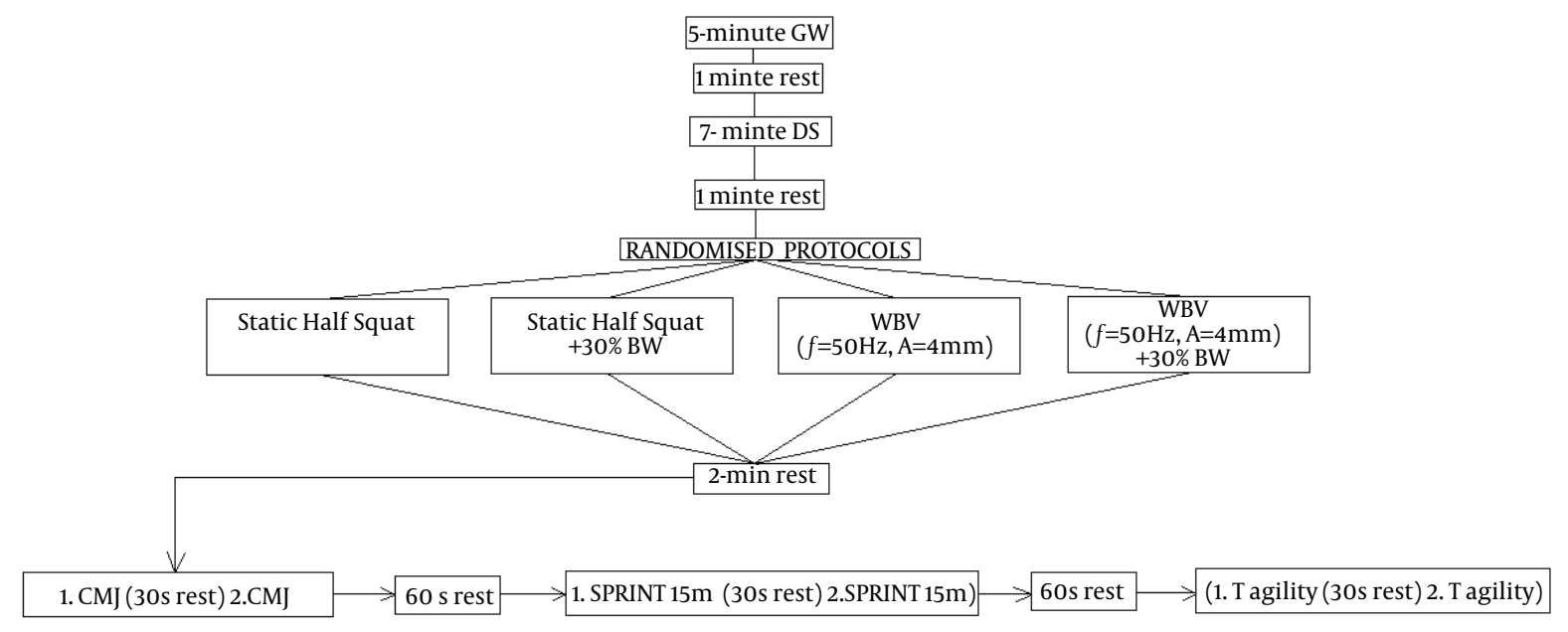

Figure 2. Experimental Design 
In the modified t-test, Mauchley's test posted violation in the assumption of sphericity, $\chi 2(5)=13.9, \mathrm{P}=0.017$. Thus, degrees of freedom were corrected using Greenhouse-Geisser estimates of sphericity at $\varepsilon=0.67$ (27). One way repeated ANOVA determined a significant difference in modified t-test time, $\mathrm{F}(2.01,40.1)=21.0, \eta 2=0.954, \mathrm{P}$ $=0.000$. Post hoc demonstrated that WBV showed lower times than $\mathrm{ST}(\mathrm{P}=0.013)$. Also, $\mathrm{WBV}+30 \% \mathrm{BW}$ posted lower times compared to $\mathrm{ST}(\mathrm{P}=0.000), \mathrm{ST}+30 \%(\mathrm{P}=0.000)$ and $\mathrm{WBV}(\mathrm{P}=0.003)$. Figure 5 depicts the agility times under various experimental protocols.

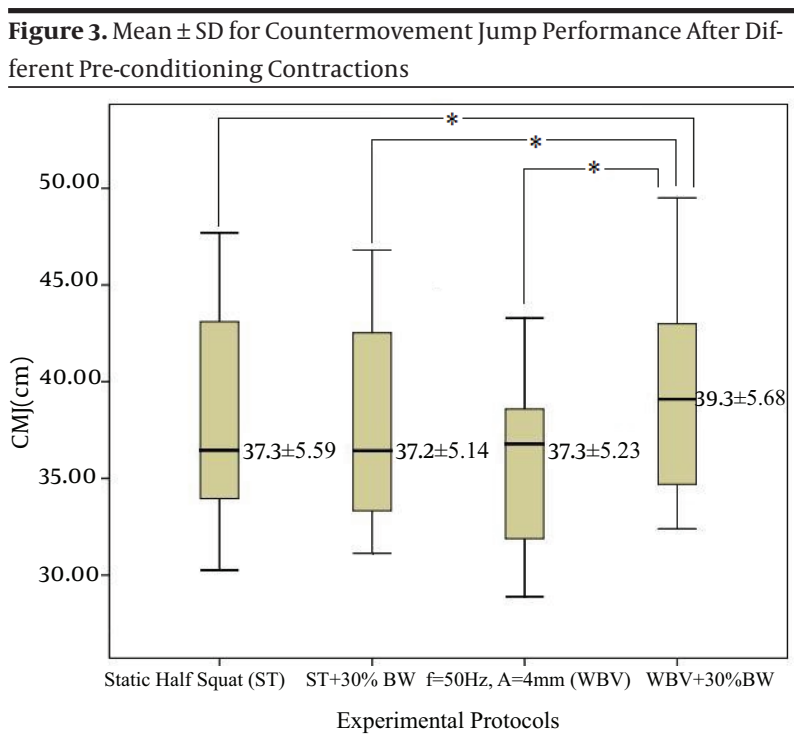

Static half squat(ST), static half squat plus $30 \%$ of body weight(ST $+30 \% \mathrm{BW}$ ) whole body vibration stimulus with $\mathrm{f}=50 \mathrm{~Hz} ; \mathrm{A}=4 \mathrm{~mm}$ (WBV), whole body vibration stimulus with $\mathrm{f}=50 \mathrm{~Hz} ; \mathrm{A}=4 \mathrm{~mm}$ and additional load of $30 \%$ of body weight (WBV $+30 \% \mathrm{BW}) .{ }^{*}$ Values significantly different from those obtained by the $\mathrm{WBV}+30 \% \mathrm{BW} ; \mathrm{P}<0.05$.

Figure 4. Mean \pm SD for Sprint Performance After Different Pre-conditioning Contractions

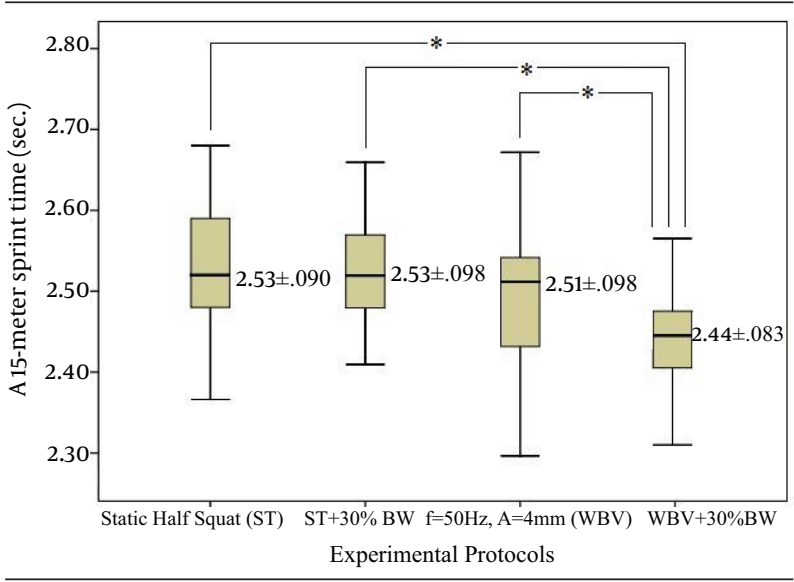

Static half squat (ST), static half squat plus $30 \%$ of body weight (ST $+30 \%$ $\mathrm{BW}$ ), whole body vibration stimulus with $\mathrm{f}=50 \mathrm{~Hz} ; \mathrm{A}=4 \mathrm{~mm}$ (WBV), whole body vibration stimulus with $\mathrm{f}=50 \mathrm{~Hz} ; \mathrm{A}=4 \mathrm{~mm}$ and additional load of $30 \%$ of body weight (WBV $+30 \% \mathrm{BW}) .{ }^{*}$ Values significantly different from those obtained by the WBV $+30 \% \mathrm{BW} ; \mathrm{P}<0.05$.
Figure 5. Mean \pm SD for Agility Performance After Different Pre-conditioning Contractions

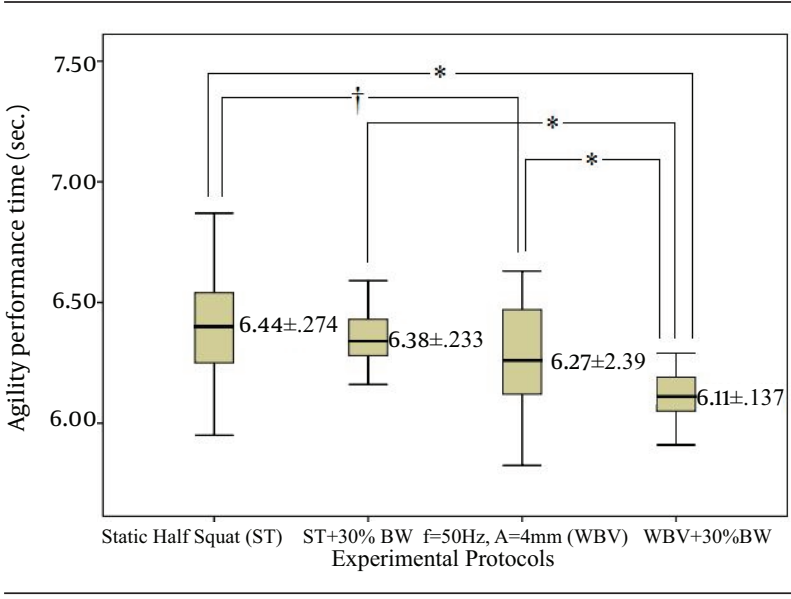

Static half squat (ST), static half squat plus $30 \%$ of body weight (ST $+30 \%$ $\mathrm{BW}$ ), whole body vibration stimulus with $\mathrm{f}=50 \mathrm{~Hz} ; \mathrm{A}=4 \mathrm{~mm}$ (WBV), whole body vibration stimulus with $\mathrm{f}=50 \mathrm{~Hz} ; \mathrm{A}=4 \mathrm{~mm}$ and additional load of $30 \%$ of body weight (WBV $+30 \% \mathrm{BW}) .{ }^{*}$ Values significantly different from those obtained by the WBV $+30 \% \mathrm{BW} ; \mathrm{P}<0.05$. †Values significantly different from those obtained by the WBV; $\mathrm{P}<0.05$.

\section{Discussion}

The purpose of this study was to compare leg power, speed and agility exhibited after loaded and unloaded static squat exercise during vibration and non-vibration conditions in male college football players. The main finding of the study showed WBV protocol with additional load of $30 \%$ of BW produced the largest gains in CMJ, speed and agility. In addition, WBV treatment showed positive effects on the physical performance compared to control preconditioning protocols without vibration stimulus.

There are no other studies that identified the effects of loaded WBV on jump, sprint and agility performance. It is quite hard to compare the results of this study to some recent studies because of methodological parameter differences. In the current study, intermittent $(5 \times 60$ seconds $)$ WBV protocol with a 30-second rest ratio was used with high frequency $(50 \mathrm{~Hz})$ and medium amplitude $(4 \mathrm{~mm})$. Most of the similar available studies utilized continued WBV protocols (30-90 seconds) in combination with low/ high frequencies/amplitudes (28-30) or intermittent protocols, but with smaller number of applied bouts of the vibration stimulus $(10,13-15)$.

In jumping, WBV $+30 \%$ BW showed greatest gains on CMJ than WBV, ST $+30 \%$ BW and ST protocols. No significant differences between WBV, ST $+30 \%$ BW and ST protocols were observed. This is somewhat in line with the findings of Adams et al. (2009) which demonstrated those high frequencies ( $40 \mathrm{~Hz}$ and $50 \mathrm{~Hz}$ ) were most effective on CMJ performance when applied in combination with high amplitudes (4-6 $\mathrm{mm}$ ) and evaluated between first and fifth minute post treatment (28). Also, 
Armstrong et al. (30) showed significant improvement in countermovement jump height after WBV at 5 minutes and 10 minutes post treatment from varying frequencies $(30,35,40$ or $50 \mathrm{~Hz})$ and amplitudes $(2-4 \mathrm{~mm}$ or $4-6 \mathrm{~mm})$ in male and female college students. In contrast, the other studies $(10,15,17,18)$ showed detrimental effects or no change in vertical jump after vibration stimulus and as it was mentioned before these equivocal findings may be a result of different WBV protocols used.

The comparative results of different preconditioning protocols on sprint performance were similar to those obtained in jumping performance. $\mathrm{WBV}+30 \% \mathrm{BW}$ protocol showed superior effects on sprint performance compared to WBV, S + 30\% BW and ST. No significant differences between $\mathrm{WBV}, \mathrm{ST}+30 \% \mathrm{BW}$ and ST protocols were observed. Although not statistically significant, WBV protocol showed better effects on sprint performance compared to non-WBV protocols. This is to some extent in agreement with the studies of Ronnestad et al.(31) who reported enhancement in 40-meter sprint performance in male football players after a 30-second WBV protocol ( $\mathrm{f}$ $=50 \mathrm{~Hz} ; \mathrm{A}=3 \mathrm{~mm}$ ) compared to a control. Furthermore, WBV at a frequency of $30 \mathrm{~Hz}$ did not show any improvement in the sprint performance compared to control. In follow-up study, Ronnestad et al. (32) demonstrated an on-ice sprint performance enhancement one minute after WBV preconditioning $(\mathrm{f}=50 \mathrm{~Hz} ; \mathrm{A}=3 \mathrm{~mm})$ in icehockey players. Contrastingly, several studies failed to provide evidence that acute WBV stimulus positively affect sprint performance. Gerakaki et al. (17) reported that WBV (90 seconds, 50 н z, 2 mm) did not lower a 60-meter sprint time as well as it did not affect a step length and rate. Cochrane (2013) (14) determined that the intermittent $(5 \times 1$ minute) WBV $(26 \mathrm{~Hz}, 6 \mathrm{~mm})$ treatment with one minute rest time between; affected $1.5 \mathrm{~m}$ sprint time compared to control group, but there was not noticeable effects between pre and post conditioning sprint times. Similarly, Kavanaugh et al. (16) and Roberts et al. (33) used a single WBV bout of 30 and 60 seconds, with vibration (50 Hz and $3 \mathrm{~mm}$ ) and $26 \mathrm{HZ}$ and $4 \mathrm{~mm}$ respectively and found no significant improvement in $30 \mathrm{~m}$ sprint performance between WBV and sham. Bullock et al. (13), who used intermittent $(3 \times 60$ seconds with 180 seconds of relief period between) WBV protocol with vibration stimulus of $30 \mathrm{~Hz}$ and $4 \mathrm{~mm}$, reported no effects on $30 \mathrm{~m}$ sprint performance in international skeleton athletes. One year later, Bullock et al. (34) tried to elicit acute potentiation with higher frequency ( $45 \mathrm{~Hz}$ ) and shorter rest period (60 seconds) between vibration stimuli, but they obtained the results similar to the previous study.

Preconditioning protocols on agility performance demonstrated significant differences between the WBV $+30 \%$ BW and other protocols. Additionally, WBV showed significant difference on agility compared to ST. Despite the widespread use and popularity of WBV and importance of agility in team sports, there is limited number of studies that investigated the acute effects of WBV on agility performance. Cochrane (14) investigated the effect of the intermittent $(5 \times 1$ minute; with 1 minute rest) WBV proto$\operatorname{col}(26 \mathrm{~Hz}, 6 \mathrm{~mm})$ on reactive agility. The results showed no significant changes for the reactive agility test. Concomitant findings were presented by Pienaar (35) which included WBV protocol before dynamic hockey-specific warm-up trying to investigate its effects on T-agility test (ATT) performance. The study used two sets of five WBV exercises with the first set using a low amplitude (2-4 mm) for 30 seconds. The second was increased to a higher amplitude (4-6 mm) and duration of 45 seconds. Both sets of WBV stimulus were set at frequency of $35 \mathrm{~Hz}$. Athletes were allowed 30 seconds of relief period between exercises and two-minute rest in between sets. Although the results did not show any significant acute effect of WBV on agility performance, improvements in applied ATT time were recorded. In a similar vein, Torvinen et al. (19) also reported no significant effects of four minutes of WBV stimulus with a progressive (every 1 minute) increment in vibration frequency (from $15 \mathrm{~Hz}$ to $30 \mathrm{~Hz}$ ) on shuttle run (change of direction and agility test) measured 2 and 60 minutes after treatments. Conflicting results with the findings of the study could be explained by various agility measurement protocols. The new modified $\mathrm{T}$ agility test used in this study covers shorter distances which was different in the previous researches. It is known that leg power has strong correlation with short distance agility performance (36). This may suggest that improvement in power performance (CMJ) also affected the results in the modified $\mathrm{T}$ agility by generating leg power in a short period of time.

Performance gains in CMJ, sprint and agility after WBV with additional load can be attributed to an increase in muscle temperature (37) and blood flow (38). It is well documented that the higher muscle temperature increases nerve conduction velocity, elevates muscle enzyme activity, increases dilatation of blood vessels and blood flow to the activated muscles that provides better muscle oxygenation during work (39-43). In a study by Rittweger et al. (44), they found out that application of $40 \%$ of external load with bodyweight under WBV increased specific oxygen uptake when compared to bodyweight WBV alone. Specific oxygen uptake was enhanced by increased muscle kinetics. In addition, another possible explanation for the superiority of the loaded WBV (50 $\mathrm{Hz}$ and $4 \mathrm{~mm}$ ) maybe related to increased tonic vibration reflex (TVR) conditions that led to better muscle capabilities; dampening external perturbations and reducing resonance effects. During WBV the mechanical vibrations produce compensatory muscle contractions as a result of TVR via excitation of primary endings of muscle spindles and activation of alpha-motor neurons (6-8). Another possible mechanism that contributed to the results may be linked to the larger presence of post activation potentiation (PAP) at $\mathrm{WBV}+30 \% \mathrm{BW}$ compared to other interventions. PAP refers to improvement in muscle performance preceded by muscle activity which can be as- 
sessed by twitch potentiation or reflex potentiation (2). However, researchers suggested that PAP that may result by WBV may be related to twitch potentiation rather than reflex potentiation $(11,45)$. WBV was reported to increase muscle activity and the neuromuscular stimulus compared with no-vibration conditions $(6,21)$. Exercise intensity is reported to be an important factor in PAP. Lowery et al. (46) suggested that high-intensity workloads may prolong the duration of PAP, while Behm et al. (47) and Vandervoort et al. (48) reported higher magnitude of PAP with increased exercise intensity. Luo et al. (49) found out that $10 \%$ and $30 \%$ load $1 \mathrm{RM}$ increased rectus femoris, vastuslateralis, and vastusmedialis electromyographic parameters under WBV. This may suggest that the additional load could lead to the higher PAP magnitude, as well as to more increased motor unit recruitment and synchronization (50) compared to WBV without extra load and low intensity intermittent isometric protocols.

Lastly, the findings of the study support the proposition of Ronnestad (21) that additional external load in WBV may facilitate larger stimulus than the unloaded WBV especially in well trained athletes. The subjects in the current study were well trained football players with welldeveloped muscular endurance and fatigue resistance. Additional load during WBV showed beneficial for their physical performance, but it could not be purported if the protocol would be valuable for other types of athletes and to what extent. Current limitations of the study include quantification of muscle activity and body temperature measurement across interventions to facilitate better understanding of the various dose-response mechanisms in the study. The study also failed to compare WBV interventions with traditional warm-up strategies.

The findings of the study supported the hypothesis. It can be concluded that WBV stimulus with additional extra load of $30 \%$ of body weight improved jump, sprint and agility performance compared to other unloaded preconditioning protocols. In practice, this information is very important because the WBV with the additional load can be added to the regular warm up session of football players to enhance muscle activation, increase body temperature and blood flow that is to improve physical performance in acute settings. Also, this kind of WBV stimulus may be used in combination with resistance strength training in order to produce long-term effects and adaptations in the neuromuscular system. Lastly, low intensity intermittent isometric protocols can be used as an alternative warm-up strategy in situations where WBV training is unavailable.

\section{Acknowledgements}

The researchers would like to thank the participants for their kind cooperation during experimentation sessions.

\section{References}

1. Dawes J, Roozen M. Developing Agility and Quickness. NSCA - National Strength \& Conditioning Association.Champaign: Human

\section{Kinetics; 2012}

2. Hodgson M, Docherty D, Robbins D. Post-activation potentiation: underlying physiology and implications for motor performance. Sports Med.2005;35(7):585-95.

3. Robbins DW, Docherty D. Effect of loading on enhancement of power performance over three consecutive trials.J Strength Cond Res. 2005;19(4):898-902.

4. Batista MA, Ugrinowitsch C, Roschel H, Lotufo R, Ricard MD, Tricoli VA. Intermittent exercise as a conditioning activity to induce postactivation potentiation. J Strength Cond Res. 2007;21(3):83740.

5. Xenofondos A, Laparadis K, Kyranoudis A. Post-activation poten tiation: Factors affecting it and the effect on performance. Journal of Physical Education and Sport. 2010;28(3):32-8.

6. Cardinale M, Lim J. Electromyography activity of vastus lateralis muscle during whole-body vibrations of different frequencies. $J$ Strength Cond Res. 2003;17(3):621-4.

7. Di Iorio F, Cesarelli M, Bifulco P, Fratini A, Roveda E, Ruffo M. The effects of whole body vibration on oxygen uptake and electromyographic signal of the rectus femoris muscle during static and dynamic squat.J Exerc Physiol. 2012;15(5):18-31.

8. Hagbarth KE, Eklund G. Motor effects of vibratory muscle stimuli in man. In: Granit R editor. Muscular Afferent and Motor Control. Stockholm: Almqvist and Wiksell; 1965. pp. 177-86.

9. Crow JF, Buttifant D, Kearny SG, Hrysomallis C. Low load exercises targeting the gluteal muscle group acutely enhance explosive power output in elite athletes. J Strength Cond Res. 2012;26(2):43842.

10. Cochrane DJ, Stannard SR. Acute whole body vibration training increases vertical jump and flexibility performance in elite female field hockey players. BrJ Sports Med. 2005;39(11):860-5.

11. Cochrane DJ, Stannard SR, Firth EC, Rittweger J. Acute wholebody vibration elicits post-activation potentiation. Eur J Appl Physiol. 2010;108(2):311-9.

12. Guggenheimer JD, Dickin DC, Reyes GF, Dolny DG. The effects of specific preconditioning activities on acute sprint performance. J Strength Cond Res. 2009;23(4):1135-9.

13. Bullock N, Martin DT, Ross A, Rosemond CD, Jordan MJ, Marino FE. Acute effect of whole-body vibration on sprint and jumping performance in elite skeleton athletes. J Strength Cond Res. 2008;22(4):1371-4.

14. Cochrane DJ. The effect of acute vibration exercise on shortdistance sprinting and reactive agility. J Sports Sci Med. 2013;12(3):497-501.

15. Cochrane DJ, Legg SJ, Hooker MJ. The short-term effect of wholebody vibration training on vertical jump, sprint, and agility performance. J Strength Cond Res. 2004;18(4):828-32.

16. Kavanaugh AA, Ramsey MW, Williams DA, Haff GG, Sands WA Stone MH. The Acute Effect of Whole Body Vibration on 30 Meter Fly Sprint Performance in NCAA Division I Sprinters and Jumpers. J Strength Cond Res. 2011;25(3):S43-4.

17. Gerakaki $M_{E}$, Evangelidis $P_{E}$, Tziortzis $S$, Paradisis GP. Acute effects of dynamic whole body vibration in well trained track \& field sprinters. J Phys Ed Sport. 2013;13(3):270 - 277.

18. Cormie P, Deane RS, Triplett NT, McBride JM. Acute effects of whole-body vibration on muscle activity, strength, and power. $J$ Strength Cond Res. 2006;20(2):257-61.

19. Torvinen S, Kannu P, Sievanen H, Jarvinen TA, Pasanen M, Kontulainen S, et al. Effect of a vibration exposure on muscular performance and body balance. Randomized cross-over study. Clin Physiol Funct Imaging. 2002;22(2):145-52.

20. Dabbs NC, Tran TT, Garner JC, Brown LE. A brief review: Using whole-body vibration to increase acute power and vertical jump performance. Strength Cond. 2012;34(5):78-84.

21. Ronnestad BR. Acute effects of various whole-body vibration frequencies on lower-body power in trained and untrained subjects. J Strength Cond Res. 2009;23(4):1309-15.

22. Pearson SJ, Hussain SR. Lack of association between postactivation potentiation and subsequent jump performance. EurJ Sport Sci. 2013;19:1-8.

23. Tsolakis C, Bogdanis GC, Nikolaou A, Zacharogiannis E. Influence of type of muscle contraction and gender on postactivation potentiation of upper and lower limb explosive performance in 
elite fencers. J Sports Sci Med. 2011;10(3):577-83.

24. Lim JJ, Kong PW. Effects of isometric and dynamic postactivation potentiation protocols on maximal sprint performance. $J$ Strength Cond Res. 2013;27(10):2730-6.

25. Sapstead G, Duncan M. Acute Effect of Isometric Mid-Thigh Pulls on Postactivation Potentiation during Stretch-Shortening Cycle and Non-Stretch-Shortening Cycle Vertical Jumps. Med Sportiva. 2013;17(1):7-11.

26. Field AP. Discovering Statistics Using SPSS: and sex and drugs and rock ' $n$ ' roll. 3 edLondon: Publications. 2009;: Sage Publications; 2009.

27. Greenhouse SW, Geisser S. On methods in the analysis of profile data. Psychometrika.1959;24(2):95-112.

28. Adams JB, Edwards D, Serravite DH, Bedient AM, Huntsman E, Jacobs KA, et al. Optimal frequency, displacement, duration, and recovery patterns to maximize power output following acute whole-body vibration.J Strength Cond Res. 2009;23(1):237-45.

29. Bedient AM, Adams JB, Edwards DA, Serravite DH, Huntsman E, Mow SE, et al. Displacement and frequency for maximizing power output resulting from a bout of whole-body vibration. $J$ Strength Cond Res. 2009;23(6):1683-7.

30. Armstrong WJ, Grinnell DC, Warren GS. The acute effect of wholebody vibration on the vertical jump height. J Strength Cond Res. 2010;24(10):2835-9.

31. Ronnestad BR, Ellefsen S. The effects of adding different wholebody vibration frequencies to preconditioning exercise on subsequent sprint performance. J Strength Cond Res. 2011;25(12):330610.

32. Ronnestad BR, Slettalokken G, Ellefsen S. Adding whole body vibration to preconditioning exercise increases subsequent onice sprint performance in ice-hockey players.J Strength Cond Res. 2013.

33. Roberts B, Hunter I, Hopkins T, Feland B. The Short-Term Effect of Whole Body Vibration Training on Sprint Start Performance in Collegiate Athletes. Int JExerc Sci. 2009;2(4):264-8.

34. Bullock N, Martin DT, Ross A, Rosemond D, Jordan MJ, Marino FE. An acute bout of whole-body vibration on skeleton start and 30-m sprint performance. European Journal of Sport Science. 2009;9(1):35-9.

35. Pienaar C. The acute effect of whole body vibration (WBV) training on power-related measurements of field hockey players: biokinetics and sport science. Afr J Phys Health Educ Recr. 2010;16(4):594-604.

36. Negrete R, Brophy J. The relationship between isokinetic open and closed chain lower extremity strength and functional performance.J Sport Rehabil. 2000;9(1):46-61.

37. Cochrane DJ, Stannard SR, Sargeant AJ, Rittweger J. The rate of muscle temperature increase during acute whole-body vibration exercise. EurJAppl Physiol. 2008;103(4):441-8.

38. Kerschan-Schindl K, Grampp S, Henk C, Resch H, Preisinger E, Fialka-Moser V, et al. Whole-body vibration exercise leads to alterations in muscle blood volume. Clin Physiol. 2001;21(3):377-82.

39. Bergh U, Ekblom B. Influence of muscle temperature on maximal muscle strength and power output in human skeletal muscles. Acta Physiol Scand. 1979;107(1):33-7.

40. Davies CT, Young K. Effect of temperature on the contractile properties and muscle power of triceps surae in humans. J Appl Physiol Respir Environ Exerc Physiol. 1983;55(1 Pt 1):191-5.

41. Hedrick A. Exercise physiology: physiological responses to warm-up. J Strength Cond Res. 1992;14(5):25-7.

42. Starkie RL, Hargreaves M, Lambert DL, Proietto J, Febbraio MA. Effect of temperature on muscle metabolism during submaximal exercise in humans. Exp Physiol. 1999;84(4):775-84.

43. Gray SC, Devito G, Nimmo MA. Effect of active warm-up on metabolism prior to and during intense dynamic exercise. Med Sci Sports Exerc. 2002;34(12):2091-6.

44. Rittweger J, Ehrig J, Just K, Mutschelknauss M, Kirsch KA, Felsenberg D. Oxygen uptake in whole-body vibration exercise: influence of vibration frequency, amplitude, and external load. Int $J$ Sports Med. 2002;23(6):428-32.

45. McBride JM, Nuzzo JL, Dayne AM, Israetel MA, Nieman DC, Triplett NT. Effect of an acute bout of whole body vibration exercise on muscle force output and motor neuron excitability. J Strength Cond Res. 2010;24(1):184-9.

46. Lowery RP, Duncan NM, Loenneke JP, Sikorski EM, Naimo MA, Brown LE, et al. The effects of potentiating stimuli intensity under varying rest periods on vertical jump performance and power. J Strength Cond Res. 2012;26(12):3320-5.

47. Behm DG, Button DC, Barbour G, Butt JC, Young WB. Conflicting effects of fatigue and potentiation on voluntary force.J Strength Cond Res. 2004;18(2):365-72.

48. Vandervoort AA, Quinlan J, McComas AJ. Twitch potentiation after voluntary contraction. Exp Neurol. 1983;81(1):141-52.

49. Luo J, Clarke M, McNamara B, Moran K. Influence of resistance load on neuromuscular response to vibration training.J Strength Cond Res. 2009;23(2):420-6.

50. Cochrane DJ. The potential neural mechanisms of acute indirect vibration. J Sports Sci Med. 2011;10(1):19-30. 